The Review of Finance and Banking

print ISSN 2067-2713, online ISSN 2067-3825

Volume 12, Issue 1, Year 2020

http://dx.doi.org/10.24818/rfb.20.12.01.06, Pages 79-95

\title{
MULTIVARIATE VAR: A ROMANIAN MARKET STUDY
}

\author{
ANDREI RUSU
}

\begin{abstract}
This paper proposes a method of estimating Value-at-Risk by combining asymmetric multivariate GARCH models and filtered historical simulation (Barone-Adesi et al., 1999). Next, incremental VaR is implemented in order to decompose the portfolio and assess the risk of every individual component. Ten competitive models were estimated and subsequently back tested using five techniques. All methodologies were applied on a sample of 11 financial assets from Bucharest Stock Exchange between 2014-07-08 and 2019-10-04. The results indicate that the method using filtered historical simulation in combination with multivariate GARCH models that account for asymmetry of financial returns lead to good VaR estimates. The methods discussed in this paper could help an investor to create a better risk-optimized portfolio, but could also be used by a regulatory authority in order to impose restrictions regarding risk..
\end{abstract}

\section{IntRoduction}

Value-at-Risk (VaR) is one of the risk measures that was and still is in the center of attention for both researchers and financial entities. Ever since VaR entered in the literature (one of the early VaR methodologies can be found in Morgan, 1996) there has been a constant tendency to improve it. As Artzner et al. (1999) showed, VaR is not a coherent measure in comparison to expected shortfall because it fails to be subadditive. However, Daníelsson et al. (2012) showed that the property of subadditivity is less likely to be violated in the tail region of the financial returns distribution when $\mathrm{VaR}$ is estimated using semi-parametric techniques in combination with elements from extreme value theory. Recently, Silahli et al. (2019) compare parametric and non-parametric methods of estimating VaR and propose an improved VaR based on Weibull distribution. They used data from the cryptocurrency market. Banihashemi and Navidi (2017) compare VaR measures and introduce a Mean-conditional VaR methodology which seems to have better performance. Other studies such as Lu et al. (2014) and Al Janabi et al. (2019) make use of copulas for VaR estimation, the latter also accounting for liquidity. The two studies were focused on the oil and commodities markets respectively. Babat et al. (2017) used VaR in an attempt to optimize the weights allocation in a portfolio of financial assets. They obtained a "near-optimal" portfolio.

Despite the relevant findings of the above studies, none of them estimated VaR using the filtered historical simulation introduced by Barone-Adesi et al. (1999) in a multivariate environment and the asymmetric version of dynamic conditional correlation (aDCC) proposed by Cappiello et al. (2006), let alone the combination of the two. In our knowledge, this area has not yet been covered by any other study; therefore, this paper aims to fill this small gap in the literature by making use of the aDCC and also combining it with the filtered historical simulation in a rolling window manner. Most studies of this type were conducted on developed or emerging markets. The present study was carried out on a portfolio selected form the

Received by the editors February 13, 2020. Accepted by the editors June 15, 2020.

Keywords: Value at Risk, Incremental VaR, Multivariate GARCH, Risk contribution.

JEL Classification: C58, G11, G17.

Andrei Rusu, PhD Student, Department of Statistics Forecasts Mathematics, Babeş-Bolyai University, ClujNapoca, 400591, Romania. E-mail: rusu.andrei24@gmail.com.

This paper is in final form and no version of it will be submitted for publication elsewhere. 
Romanian stock market which, according to FTSE Russel classification, is still considered a frontier market.

Last, but not least incremental VaR as in Mina and Xiao (2001) was used in order to decompose the portfolio risk and to emphasize the risk contribution of every component.

The present work is structured in five sections: The second section provides a review of studies that focus on the VaR measure, the third section describes the data sample and other data related issues; a description of all VaR methodologies applied can also be found in this section; section number four presents findings, empirical results and comparisons with related research from the literature, and the last section concludes the study.

Note that in this paper the symbol ' denotes transposition.

\section{Literature REVIEW}

Value at risk is a measure that enjoys a high degree of popularity among financial entities and researchers. However, it is always challenged by the competing measure Expected shortfall (ES) also known as expected tail loss (ETL). One of the main disadvantages of VaR is the fact that it does not offer a clear image of what the loss will be if the $\alpha$-quantile threshold is exceeded, while expected shortfall provides such a result. Another disadvantage of VaR consists of it not being a coherent measure in the way Artzner et al. (1999) showed, meaning that it does not always satisfy the property of being subadditive, while the expected shortfall does. Studies like Rau-Bredow (2002) and Yamai and Yoshiba (2002) present comparative studies of the two risk measures. The former research focuses on the calculation of marginal risk contributions using the two measures. The study highlights the disadvantages of VaR (mainly the lack of subadditivity) and proposes the Expected Shortfall as an alternative, but as its author stated that the results' quality is uncertain in the case of random variables with discrete distributions. Yamai and Yoshiba (2002) also compare the VaR and ES with respect to three areas: the first one is the errors resulting from the estimation process; the second is risk factors decomposition and last but not least, the optimization problem. This paper also favors Expected Shortfall over VaR, however ES requires a larger data sample than VaR in order to have a robust estimation, as Yamai and Yoshiba (2002) also acknowledged. Moreover, the study is inclined towards a more theoretical-to-simulation approach rather than a practical application. Despite all the above mentioned arguments in favor of ES, VaR is less likely to fail being subadditive if it is estimated via semi-parametric methods combined with concepts from extreme value theory as Daníelsson et al. (2012) pointed out. In their study, Kellner and Rösch (2016) compare VaR and ES from a regulatory point of view and their findings indicate that expected tail loss offers a higher potential for regulatory arbitrage than value-at-risk, but its risk of parameter misspecification is also higher.

Given the pros and cons of both VaR and ES, a couple of papers focused on developing methods of estimating or enhancing both measures. Glasserman (2005) studied the decomposition of a credit risk portfolio, using importance sampling techniques (see Glasserman and Li, 2005, for details) for value at risk and expected shortfall. The methods are implemented in a Gaussian copula approach. The article proposes a complex methodology for the estimation of the two measures on the one hand, but on the other hand data for this domain is unavailable in most cases due to secrecy of banks. Krause and Paolella (2014) propose a method for predicting VaR and ES which takes into account asymmetric information, leptokurtosis and heteroskedasticity. They use a combination of GARCH family models and skewed $t$ innovations, resulting in high accuracy outcomes even for a small sample. Taylor (2020) uses both measures in a joint estimation framework. Specific scoring functions are used in order to allocate different weights to VaR and ES in order to forecast the loss. The results of the joint measures outperform the ones obtained from using the individual methods. Another study that makes use of a joint estimation of value-at-risk and expected shortfall is the one of Le (2020). The author uses a Mixed Data Sampling (called MIDAS) framework in order to make predictions over multi-day horizons. Its findings suggest that the mixed data approach has a higher degree of accuracy 
than traditional methods such as the ones based on GARCH-family models. Su et al. (2020) developed a back testing procedure for both conditional VaR and conditional ES. Based on a simulation approach, the authors prove that the new test has a good performance on a finite sample, while being robust to fat tails.

Another category of studies focuses on methodologies for VaR estimation. One of the most comprehensive papers is the one of Abad et al. (2014). It offers a compendium of Value-at-Risk methodologies developed up to the year 2014 and various ways of back testing, from which some are also used in this paper. But their study is focused more on estimation of VaR for univariate series rather than portfolios. The study is nevertheless a valuable contribution to the literature and a good starting point for any research in this domain. Halkos and Tsirivis (2019) did a comprehensive review of portfolio VaR methodologies on the energy commodities markets. In their study, the authors, summarize a series of papers from 2003 to 2017 and compared the results. Their conclusion was that on the energy market there is no VaR model that outperforms all others. Referring to different VaR methodologies, a comparative study was performed by Silahli et al. (2019). Their research is similar to the one found in the current paper, having compared different portofolio VaR models based on the variance-covariance approach, multivariate GARCH and historical simulation. A two-sided Weibull distribution VaR model is proven by the authors to perform well on cryptocurrency market. An application of their methodology on other markets could have also been considered.

Some papers studied the decomposition of value-at-risk. Hallerbach (2002) acknowledges a financial entity's need for VaR decomposition and attempts to overcome the restrictions imposed by assumptions of normality by proposing an approach based on simulation. The author finds an accurate estimator that is also efficient from a computational point of view, but the method was tested only on options contracts. An evaluation of its performances on other types of financial assets could have also been considered. A method of decomposing value at risk and expected shortfall was proposed by Boudt et al. (2008). The technique was based on asymptotic expansions and it was applied on alternative investments portfolio. Their method takes into account asymmetric information of financial returns, but all results are obtained under the hypotheses of continuous return distribution and constant conditional portfolio moments.

Other studies take into account the impact of shocks in the economy and optimal allocation of financial assets. Kim et al. (2015) introduce a new tool for calculating VaR in a multivariate environment by using a combination autoregressive vectors and quantile based models. The study was conducted on 230 financial institutions worldwide, during January 2000 - August 2010. Their study proposes a complex and advanced methodology which help analyze the impact that shocks in the economy have on financial institutions such as banks and insurance companies. The method can also be used for stress testing by a financial entity or by the regulatory authorities. It does not include however the VaR decomposition. Martellini and Ziemann (2010) focus on higher order moments when it comes to techniques of optimal selection of assets in a portfolio. This method is aimed at reducing the number of parameters involved so that less data is needed for a robust estimation. Their findings suggest that the enhanced estimates of coskewness and cokurtosis have a good performance. Jain and Chakrabarty (2019) use a multivariate approach to estimate a marginal form of $\mathrm{VaR}$ in order to determine if a financial asset is desirable in a managed portfolio. One positive aspect of their methodology is the fact that it takes into account systematic (market) risk when estimating VaR, not just the risk of the analyzed financial asset. Their findings suggest that if an entity is using this type of marginal VaR in order to select the assets to be included in the portfolio, then that portfolio will perform better.

In this study, the focus was set on estimating value at risk for a portfolio of financial assets by the use of filtered historical simulation as in Barone-Adesi et al. (1999) and the multivariate GARCH models estimated with methodologies proposed by Engle and Sheppard (2001) and Cappiello et al. (2006). Also VaR is decomposed in order to assess the individual contribution of every stock to the overall risk. 


\section{Data And Methodology}

For this study, a sample of 11 stocks was selected from the Romanian market and also included in the BET index. Their symbols are FP, TLV, SNP, SNG, BRD, TGN, EL, SNN, TEL, COTE and BVB. For the detailed list of the companies, please see Appendix 1. Initially, the research was meant to include all 16 stocks that are currently included in the BET index, at Bucharest Stock Exchange. However, due to lack of data, five stocks were eliminated. The BET index is the reference index on the Romanian market and it is composed of the most liquid stocks on the market. The data set contains information between 2014-07-08 and 2019-10-04, resulting in 1319 daily returns per stock.

The procedures used and described below, require a complete dataset. Therefore, the missing data were filled with the average price of the five days before and five days after with respect to the missing daily price. However, the impact of the selected approach is minimal due to a tiny number of missing values. ${ }^{1}$ Also, the procedure was tested with different window lengths and the results remained the same. Then, the daily returns were calculated.

All the data was collected from the website https://www.investing.com. Also, in this study, the portfolio was composed using equal weights of every stock. An equal weighted portfolio might have the advantage of favoring small companies (which might yield higher returns), but also the associated risk will be greater. Equal weighting was used in this study in order to highlight the different contributions of risk in terms of incremental VaR and also for simplicity reasons.

In order to estimate $\mathrm{VaR}$, ten methods were selected: the first six are parametric and the last four come from the semi-parametric area. Next, the total VaR is decomposed in individual contributions for every stock. Then every method is back tested using a set of five tests.

The first method is based on the Riskmetrics (Morgan, 1996) portfolio theory methodology and assumes the normality of the financial returns. VaR would be determined in the following way:

where:

$$
\operatorname{VaR} R_{\alpha}=\mu+z_{\alpha} \sigma
$$

(1) $\mu$ is the mean of the portfolio return series;

(2) $z_{\alpha}$ is the standard normal distribution $\alpha$-quantile.

(3) $\sigma$ represents the portfolio standard deviation, which is determined as:

$$
s=\sqrt{w^{\prime} \Sigma w}
$$

where:

(1) $\Sigma$ is the variance-covariance matrix of the portfolio components;

(2) $w$ is a vector containing the weight of every stock in the portfolio.

This method is the most popular among financial entities due to its simplicity, which is why it was chosen as a benchmark for this study.

The second method is based on the one proposed by Favre and Galeano (2002) which determine VaR using a Cornish - Fisher expansion approach. The authors called it "Modified value at risk". Their formula for a univariate financial return series is the following:

$V a R_{\alpha}=\omega\left[\mu-\left(z_{\alpha}+\frac{1}{6}\left(z_{\alpha}^{2}-1\right) S k+\frac{1}{24}\left(z_{\alpha}^{3}-3 z_{\alpha}\right) K u-\frac{1}{36}\left(2 z_{\alpha}^{3}-5 z_{\alpha}\right) S k^{2}\right) \sigma\right]$

where:

(1) $\omega$ is the amount of money invested in a specific stock;

(2) $\mu$ is the mean of the financial returns of the previously mentioned stock;

\footnotetext{
${ }^{1}$ Missing values: SNG $-1(0.08 \%), \mathrm{EL}-2(0.15 \%), \mathrm{SNN}-3(0.23 \%), \mathrm{TEL}-1(0.08 \%)$, COTE $-30(2.27 \%)$, BVB $-8(0.63 \%)$. For the rest of the stocks, there were no missing values.
} 
(3) $\sigma$ denotes the standard deviation of the financial returns;

(4) $z_{a}$ represents the quantile from the standard normal distribution at confidence level $\alpha$;

(5) $S k$ and $K u$ are the skewness and kurtosis of the financial return series, respectively.

This method has the advantage of taking into account the asymmetry of the financial returns. For details about how to estimate the portfolio VaR based on equation (3), please see Boudt et al. (2008). For simplicity, in this study it was assumed that the amount of money invested in every stock is one monetary unit.

The next methods are parametric methods and are based on letting the variance covariance matrix change over time, instead of assuming it constant. They are based on multivariate GARCH family models with dynamic conditional correlation (DCC) as proposed by Engle and Sheppard (2001) and asymmetric dynamic conditional correlation Cappiello et al. (2006).

In the case of the DCC, the authors propose a two step method: at the first step, a univariate GARCH model is fit on every financial asset series. Then, at the second step the process continues with the fitting of the multivariate model with the following correlation structure:

$$
Q_{t}=\left(1-\sum_{m=1}^{M} a_{m}-\sum_{n=1}^{N} b_{n}\right) \bar{Q}+\sum_{m=1}^{M} a_{m}\left(\varepsilon_{t-m} \varepsilon_{t-m}^{\prime}\right)+\sum_{n=1}^{N} b_{n} Q_{t-n}
$$

where:

(1) $\bar{Q}$ is the unconditional covariance between standardized errors which was estimated in the first step of the process;

(2) $\varepsilon_{t-m}$ are "residuals standardized by their conditional standard deviation" as Engle and Sheppard (2001) explain;

(3) $a_{m}$ and $b_{n}$ represent parameters to be estimated.

In the case of aDCC model, Cappiello et al. (2006) propose a modified version of the dynamic correlation model that takes into consideration the asymmetric information. The model is described by the following equation:

$$
Q_{t}=\left(\bar{P}-\alpha^{2} \bar{P}-\beta^{2} \bar{P}-\gamma^{2} \bar{N}\right)+\alpha^{2} \epsilon_{t-1} \epsilon_{t-1}^{\prime}+\gamma^{2} n_{t-1} n_{t-1}^{\prime}+\beta^{2} Q_{t-1}
$$

where

(1) $\bar{P}=E\left(\epsilon_{t} \epsilon_{t}^{\prime}\right)$;

(2) $\bar{N}=E\left(n_{t} n_{t}^{\prime}\right)$;

(3) $n_{t}=I\left[\epsilon_{t}<0\right] \circ \epsilon_{t}$;

(4) The sign $\circ$ denotes the element-wise product, $I[]$ is an indicator function that takes the value of 1 when the condition $\epsilon_{t}<0$ is satisfied and 0 otherwise;

(5) $\epsilon_{t}$ represents standardized residuals;

(6) $\alpha, \beta$ and $\gamma$ are the model parameters.

The covariance matrices obtained with the above presented models were used to estimate VaR on a daily basis in a similar manner with the one presented in Riskmetricks. The difference is that the variance-covariance matrix was different for every day, resulted from the dynamic correlation models.

Four models of this type were chosen: a GARCH model as in Bollerslev (1986) in combination with both DCC and aDCC in a multivariate approach and an APARCH model as proposed by Ding et al. (1993) combined with the same two multivariate approaches mentioned above.

The last four models are based on a semi-parametric approach. They use the combination of the above explained multivariate methods, DCC and aDCC, and the filtered historical simulation (FHS) introduced by Barone-Adesi et al. (1999). This method consists of four main steps: first, one must fit a model for conditional volatility on every return series in the portfolio. The authors recommended an asymmetric GARCH-family model. Then the realized returns must be computed by dividing the observed return to the estimated volatilities. The third step consists of bootstrapping with replacement a large enough number of returns from the ones 
obtained at the second step. The extracted returns will then be multiplied with the one day ahead forecasted volatility. Then, at the last step, $\operatorname{VaR} \alpha$ is extracted from the sample obtained at the third step, at the chosen $\alpha$-quantile.

In the multivariate case the portfolio return needs to be divided by the portfolio volatility. First, a univariate GARCH-family model must be chosen, in order to fit the univariate series. Then the process continues using one of the two approaches described above, DCC or aDCC. Then the variance-covariance matrixes become available.

The Riskmetricks approach was used in order to obtain the simple portfolio returns in the following manner:

$$
R_{p f, t}=\sum_{i=1}^{N} w_{i} r_{i t}
$$

where:

(1) $R_{p f, t}$ is the portfolio return at time $t$;

(2) $r_{i t}$ is the return of stock $i$ at time $t$;

(3) $w_{i}$ represents the weight of stock $i$ in the portfolio.

The portfolio volatility $\sigma_{p f, t}$ was determined in the following way:

$$
\sigma_{p f, t}=\sqrt{w^{\prime} \Sigma_{t} w}
$$

where:

(1) $\Sigma_{t}$ is the portfolio variance-covariance matrix at time $t$ obtained from DCC and aDCC models;

(2) $w$ is a vector containing the weights of every stock in the portfolio.

Having obtained both the portfolio returns and volatilities, the realized portfolio returns can be computed as described previously and the FHS approach can be implemented. In this study, 4 models have been chosen based on this approach. The first two use a GARCH model introduced by Bollerslev (1986) in combination with DCC and aDCC methods, and the last two the APARCH proposed by Ding et al. (1993), combined with the same two multivariate methods.

In modeling the volatility of the financial returns time series, two of the most popular and competing categories of models are the GARCH models and the stochastic volatility models. Hafner and Preminger (2010) developed a procedure that chooses the best approach from the two previously mentioned. They tested the methodology on simulated data and observed data and concluded that the GARCH model was chosen in most cases. Another reason for which GARCH was used consists in the simplicity of implementation.

In order to make the back testing possible, all the above mentioned approaches were used in a rolling window manner. Given the fact that the whole sample had a length of 1319 trading days, the window was selected to have 500 days length, thus remaining with a length 819 days as the testing sample. In the filtered historical simulation approaches the bootstrap sample had a length of 300 observations.

Five back testing methods were used in order to determine the VaR accuracy: the failure rate,, the unconditional coverage test proposed by Kupiec (1995), the conditional coverage test introduced by Christoffersen (1998), the dynamic quantile (DQ) test of Engle and Manganelli (2004) and the average quantile loss function as found in Gonzalez-Rivera et. al (2004).

The failure rate is determined by simply computing the ratio of the number of financial returns that exceed the $\mathrm{VaR}$ divided by the total number of returns in the corresponding window. If the model predicts $\mathrm{VAR}_{\alpha}$ correctly the fail rate should have a value around the chosen threshold $\alpha$.

Kupiec (1995) proposed a test known in literature as the unconditional coverage test. The test compares the proportion of exceptions above VaR that occur in a financial return series 
with the significance threshold $\alpha$ at which VaR is determined, in order to see if there is any difference between the two from a statistical point of view. The likelihood ratio $L R^{u c}$ of the test is:

$$
L R^{u c}=2\left[\log \left((1-\widehat{\alpha})^{n_{0}} \widehat{\alpha}^{n_{1}}\right)-\left((1-\alpha)^{n_{0}} \alpha^{n_{1}}\right)\right]
$$

which follows a chi-square distribution.

(1) $n_{0}$ is the number of non-exceptions;

(2) $n_{1}$ represents the number of exceptions;

(3) $\alpha$ is the threshold at which VaR is calculated;

(4) $\widehat{\alpha}$ is the number of exceptions divided by the total number of observations.

The test known as the conditional coverage test was introduced by Christoffersen (1998) and it is based on the unconditional coverage test, with the exception that it takes into account the serial correlation present in the financial return series. The likelihood ratio $L R^{c c}$ of this test is the following:

$$
L R^{c c}=2\left[\log \left(\left(1-\hat{p}_{01}\right)^{n_{00}} \hat{p}_{01}^{n_{01}}\left(1-\hat{p}_{11}\right)^{n_{10}} \hat{p}_{11}^{n_{11}}\right)-\log \left((1-\alpha)^{n_{0}} \alpha^{n_{1}}\right)\right]
$$

which also follows a chi-square distribution:

(1) $n_{0}$ and $n_{1}$ have the same significance as in equation (8);

(2) $n_{i j}$ represents the total number of transitions that occur from state $i$ to state $j$;

(3) $\hat{p}_{i j}$ is the probability of transition from state $i$ to state $j$ between two consecutive observations, in a Markov chain manner;

(4) the states $i$ and $j$ can take a value of 0 or 1 meaning that an observation is a correctly predicted VaR or an exception and parameter $\alpha$ represents the VaR threshold.

The null hypotheses of the conditional and unconditional correlation tests are that the VaR model correctly predicts the series of returns.

Another test used is the one introduced by Engle and Manganelli (2004), also known as the dynamic quantile test which will be denoted in the latter by $D Q$. The authors defined the Hit function such that it will take a value $1-\alpha$ when the return is less than the $\operatorname{VaR}$ and $-\alpha$ otherwise. The $D Q$ statistic follows a chi-square distribution and it is given by:

$$
D Q=\frac{N^{-1} H i t^{\prime} X\left[X^{\prime} X\right]^{-1} X^{\prime} H i t}{\alpha(1-\alpha)}
$$

where:

(1) $N$ is the number of the out of sample observations;

(2) Hit is a vector containing the values of the function mentioned above;

(3) $\alpha$ is the VaR significance level;

(4) $X$ is a matrix which contains lagged values of $H i t$, lagged values of the financial return series, and VaR values. For additional details, please see Engle and Manganelli (2004).

The quantile loss function $Q$ of Gonzalez-Rivera et. al (2004) is also reported. The function is defined by the relation:

$$
Q=\sum_{t=R}^{T}\left(\alpha-I_{t+1}^{\alpha}\right)\left(r_{t+1}-V a R_{t+1}^{\alpha}\right)
$$

where:

(1) $I_{t+1}^{\alpha}$ takes the value of 1 if $r_{t+1}<V a R_{t+1}^{\alpha}$ and 0 otherwise;

(2) $r_{t+1}$ is the financial return at time $t+1$;

(3) $\alpha$ is the significance threshold for VaR. 
A lower value of $Q$ means a better fit model.

Last but not least, a portfolio decomposition method was applied in order to obtain the contribution of every stock analyzed to the portfolio value at risk. This is one method proposed by Mina and Xiao (2001) and it is known as incremental VaR. According to the authors, this measure provides information about how the portfolio will look like if an investor would modify the structure of his/her portfolio by small amounts. The incremental value at risk is calculated in the following manner:

$$
\text { Inc.VaR }=w\left(-\mu-z_{\alpha} \frac{\Sigma w}{\sqrt{w^{\prime} \Sigma w}}\right)
$$

where:

(1) $\mu$ is the portfolio average return;

(2) $z_{\alpha}$ is the $\alpha$ - quantile extracted from the standard normal distribution;

(3) $\mathrm{w}$ is the vector of weights associated with every stock in the portfolio;

(4) $\Sigma$ is the variance-covariance matrix.

The incremental VaR calculus was done using the function $V a R$ from the PerformanceAnalytics package for the Riskmetrics and Modified VaR approaches. In R, the incremental VaR is implemented and known as component VaR.

For the parametric multivariate approaches the variance-covariance matrix $\Sigma$ was estimated on the daily basis using multivariate GARCH and APARCH specifications. As for the semiparametric methods, $\Sigma$ was estimated in the same way as in the fully parametric cases and $z_{\alpha}$ was replaced with the realized returns as described by Barone-Adesi et al. (1999).

All calculations were done in $\mathrm{R}$ environment using the following packages: PerformanceAnalytics, rmgarch, xts and GAS. For the purpose of this article, equal weights were used when the portfolio was constructed. As stated before, this allows an intuitive comparison of the different contributions of every stock to the overall risk in terms of incremental VaR. Through the course of this paper, the significance threshold $\alpha$ was considered $1 \%$ due to the fact that the value represents a standard for high rating (as in Moody, FITCH etc.) financial entities. The 5\% significance level is also a standard value, but it is used by companies with lower ratings.

\section{Results}

This section presents the empirical results obtained by analyzing the data sample. Table I below presents the descriptive statistics for the daily returns of the eleven stocks during 2014-07-08 and 2019-10-04.

\begin{tabular}{|c|c|c|c|c|c|c|c|}
\hline \multicolumn{7}{|c|}{ Table I - Summary statistics of daily returns } \\
\hline Stock & Mean & Median & Std_Dev & Min & Max & Skew & Kurt \\
\hline FP & 0.00026 & 0 & 0.01082 & -0.06970 & 0.04652 & -0.90333 & 8.62616 \\
\hline TLV & 0.00049 & 0 & 0.01705 & -0.22211 & 0.10523 & -2.79511 & 36.21542 \\
\hline SNP & -0.00010 & 0 & 0.01539 & -0.13890 & 0.10017 & -0.43585 & 9.65767 \\
\hline SNG & 0.00006 & 0 & 0.01342 & -0.15234 & 0.05166 & -2.90537 & 26.19479 \\
\hline BRD & 0.00031 & 0 & 0.01544 & -0.18172 & 0.10338 & -1.78606 & 23.51975 \\
\hline TGN & 0.00043 & 0 & 0.01358 & -0.13931 & 0.06677 & -1.87881 & 20.31259 \\
\hline EL & 0.00000 & 0 & 0.01271 & -0.10636 & 0.05987 & -0.60919 & 6.42958 \\
\hline SNN & 0.00036 & 0 & 0.01397 & -0.19106 & 0.10479 & -2.06798 & 32.80821 \\
\hline TEL & -0.00012 & 0 & 0.01332 & -0.09706 & 0.07498 & -0.77652 & 8.74013 \\
\hline COTE & 0.00036 & 0 & 0.01714 & -0.28659 & 0.13799 & -3.21237 & 67.61629 \\
\hline BVB & -0.00011 & 0 & 0.01270 & -0.07274 & 0.08038 & 0.40278 & 4.72591 \\
\hline \multicolumn{7}{|c|}{ Source: own computations in R environment } \\
\hline
\end{tabular}

Even though the means are very close to zero and the standard deviations are rather low, it is noticeable that most of the stocks have a left-asymmetric behavior compared to a normal distribution, a typical characteristic for financial asset returns series. Also, the values of 
the kurtosis show a high deviation from a normal distribution, all indicating a higher peaked empirical distribution.

Table II presents the results of the five back testing methods, failure rate the unconditional coverage test - UC (Kupiec, 1995), the conditional coverage test - CC (Christoffersen, 1998), the dynamic quantile test - DQ (Engle and Manganelli, 2004) and the Loss function (GonzalezRivera et. al, 2004) respectively.

\begin{tabular}{|c|c|c|c|c|c|}
\hline \multicolumn{7}{|c|}{ Table II - Results of VaR back testing methods } \\
\hline Model & Fail_rate & UC_Pval & CC_Pval & DQ_Pval & Loss \\
\hline Riskmetrics & $1.829 \%$ & 0.03245 & 0.07675 & 0.05993 & 0.00043494 \\
\hline Modified VaR & $0.244 \%$ & 0.00910 & 0.03316 & 0.29167 & 0.00054135 \\
\hline GARCH-DCC & $1.220 \%$ & 0.54137 & 0.73336 & 0.00270 & 0.00036479 \\
\hline GARCH-aDCC & $1.098 \%$ & 0.78221 & 0.87092 & 0.92204 & 0.00036143 \\
\hline APARCH-DCC & $1.585 \%$ & 0.12059 & 0.24305 & 0.00008 & 0.00035742 \\
\hline APARCH-aDCC & $1.220 \%$ & 0.54137 & 0.73336 & 0.78223 & 0.00034940 \\
\hline FHS-GARCH-DCC & $0.854 \%$ & 0.66573 & 0.85756 & 0.98442 & 0.00043263 \\
\hline FHS-GARCH-aDCC & $0.854 \%$ & 0.66573 & 0.85756 & 0.97139 & 0.00042622 \\
\hline FHS-APARCH-DCC & $0.732 \%$ & 0.41746 & 0.68866 & 0.88348 & 0.00042632 \\
\hline FHS-APARCH-aDCC & $0.854 \%$ & 0.66573 & 0.85756 & 0.98356 & 0.00043407 \\
\hline Source: own computations in R environment \\
\hline \multicolumn{7}{|c|}{ Note: Fail_rate=Failure rate, } \\
\hline UC_Pval = P-value of Kupiec's (1995) unconditional coverage test \\
\hline CC_Pval = P-value of Christofersen's (1998) conditional coverage test \\
\hline DQ_Pval = P-value of Dynamic Quantile test of Engle and Manganeli (2004) \\
\hline Loss = Value of the loss function of Gonzalez-Rivera et al. (2004) \\
\hline
\end{tabular}

The null hypothesis of the above tests (UC- unconditional coverage, CC - conditional coverage, DQ-dynamic quantile) is that the model predicts VaR correctly. A p-value lower than 0.05 threshold shows the rejection of this hypothesis. In case of the loss function the best model is the one with the lowest value.

In all cases, VaR was estimated at $1 \%$ significance threshold; therefore the failure rate of a model is expected to be around the value of $1 \%$. The Riskmetrics approach was considered a benchmark for this study and it also has the highest failure rate.

The Modified VaR has the lowest fail rate and one could argue that it should be considered the best method. However, it is actually overestimating the risk and it fails both the conditional and unconditional coverage tests. It also has the highest loss value which would put it on the last place according to this criterion.

Out of the pure parametric models, the best one seems to be the GARCH-aDCC. It's failure rate has the smallest difference from the threshold of $1 \%$ and it performs best at the unconditional and conditional coverage tests overall. The other parametric models tend to underestimate the risk a bit, but they also yield good results, except for the APARCH-DCC which is too far from the $1 \%$ threshold.

In the case of semi-parametric approaches, all of them tend to slightly overestimate the risk, but apart from FHS-APARCH-DCC which yielded the worst result, the other ones, perform rather well. The identical failure rate is due to the small bootstrap sample size. The window was chosen to have 500 observations and the bootstrap window length had 300 observations. The best models are the ones that used GARCH for the univariate specifications. They perform well on all 3 statistical tests and the one which takes into account* the asymmetry on the multivariate level also has the lowest loss.

As for the risk decomposition part, Table III contains summary statistics of the daily contribution series on the back testing period. Graphs with the contributions, in percentages, of every stock to the portfolio VaR, for the entire test sample are also included in Appendix 2. 
The graphs are displayed for GARCH-aDCC and FHS-GARCH-aDCC models; the rest of them can be provided at request.

The table below contains the average contribution, the median contribution, the minimum and maximum contributions, respectively for every stock, on the back testing period. For most of the stocks in all models, the mean values are above their corresponding medians which indicate the possibility of extreme values. For some stocks the maximum contributions reach very high levels, fact most visible at COTE stock. Also, the pure parametric models seem to generate higher extremes compared to their semi-parametric equivalents. This is visible in the graphs included in appendix 2. Therefore, the results shown in table III strengthen the ones from table II, namely the filtered historical simulation approaches perform better than their pure parametric counterparts.

\begin{tabular}{|c|c|c|c|c|c|c|c|c|}
\hline \multicolumn{9}{|c|}{ Table III - Results for contribution (\%) of stocks to portfolio VaR } \\
\hline & \multicolumn{4}{|c|}{ Riskmetrics } & \multicolumn{4}{|c|}{ Modified VaR } \\
\hline Stock & Mean & \multirow{2}{*}{$\begin{array}{c}\text { Median } \\
0.056\end{array}$} & \multirow{2}{*}{$\begin{array}{c}\text { Min } \\
0.037\end{array}$} & Max & \multirow{2}{*}{$\begin{array}{l}\text { Mean } \\
0.079\end{array}$} & \multicolumn{2}{|c|}{\begin{tabular}{l|l} 
Median & Min \\
\end{tabular}} & Max \\
\hline $\mathrm{FP}$ & 0.060 & & & 0.085 & & 0.082 & 0.020 & 0.124 \\
\hline TLV & 0.112 & 0.121 & 0.084 & 0.143 & 0.079 & 0.089 & -0.048 & 0.25 \\
\hline SNP & 0.108 & 0.110 & 0.088 & 0.122 & 0.098 & 0.144 & 0.012 & 0.15 \\
\hline SNG & 0.088 & 0.087 & 0.072 & 0.105 & 0.070 & 0.066 & 0.022 & 0.12 \\
\hline BRD & 0.105 & 0.109 & 0.084 & 0.132 & 0.094 & 0.063 & 0.049 & 0.21 \\
\hline TGN & 0.094 & 0.093 & 0.074 & 0.122 & 0.082 & 0.082 & 0.070 & 0.08 \\
\hline EL & 0.082 & 0.081 & 0.063 & 0.100 & 0.086 & 0.091 & 0.060 & 0.11 \\
\hline SNN & 0.086 & 0.086 & 0.067 & 0.097 & 0.033 & 0.011 & -0.02 & 0.09 \\
\hline TEL & 0.086 & 0.086 & 0.076 & 0.098 & 0.069 & 0.071 & 0.056 & 0.09 \\
\hline COTE & 0.117 & 0.105 & 0.068 & 0.170 & 0.272 & 0.065 & 0.034 & 0.66 \\
\hline BVB & 0.061 & 0.060 & 0.050 & 0.073 & 0.039 & 0.028 & -0.03 & 0.12 \\
\hline & & GARC & H_DCC & & & $\mathrm{ARCH}$ & _aDCC & \\
\hline FP & 0.055 & \begin{tabular}{|l|}
0.051 \\
\end{tabular} & 0.019 & 0.268 & 0.055 & 0.051 & \begin{tabular}{|l|}
0.021 \\
\end{tabular} & 0.264 \\
\hline TLV & 0.124 & 0.125 & 0.019 & 0.185 & 0.123 & 0.124 & 0.018 & 0.184 \\
\hline SNP & 0.112 & 0.109 & 0.028 & 0.197 & 0.112 & 0.108 & 0.031 & 0.196 \\
\hline SNG & 0.099 & 0.103 & 0.022 & 0.127 & 0.099 & 0.103 & 0.025 & 0.126 \\
\hline BRD & 0.111 & 0.107 & 0.035 & 0.354 & 0.111 & 0.106 & 0.039 & 0.343 \\
\hline TGN & 0.091 & 0.085 & 0.034 & 0.306 & 0.091 & 0.085 & 0.034 & 0.300 \\
\hline $\mathrm{EL}$ & 0.088 & 0.086 & 0.029 & 0.156 & 0.088 & 0.087 & 0.030 & 0.159 \\
\hline SNN & 0.081 & 0.070 & 0.022 & 0.423 & 0.081 & 0.069 & 0.026 & 0.422 \\
\hline TEL & 0.087 & 0.080 & 0.033 & 0.400 & 0.087 & 0.080 & 0.033 & 0.397 \\
\hline COTE & 0.084 & 0.075 & 0.033 & 0.676 & 0.084 & 0.075 & 0.033 & 0.642 \\
\hline BVB & 0.068 & 0.071 & 0.011 & 0.089 & 0.069 & 0.071 & 0.010 & 0.091 \\
\hline & & 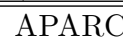 & $\overline{\mathrm{H}} \quad \mathrm{DC}$ & & & $\overline{\mathrm{PARCH}}$ & $\mathrm{IaDC}$ & \\
\hline FP & 0.058 & 0.053 & 0.025 & 0.263 & 0.058 & 0.053 & 0.023 & 0.252 \\
\hline TLV & 0.124 & 0.121 & 0.013 & 0.215 & 0.124 & 0.121 & 0.013 & 0.216 \\
\hline SNP & 0.111 & 0.109 & 0.023 & 0.193 & 0.111 & 0.108 & 0.031 & 0.190 \\
\hline SNG & 0.088 & 0.087 & 0.021 & 0.147 & 0.088 & 0.087 & 0.027 & 0.167 \\
\hline BRD & 0.109 & 0.108 & 0.016 & 0.188 & 0.108 & 0.107 & 0.024 & 0.188 \\
\hline TGN & 0.089 & 0.084 & 0.048 & 0.322 & 0.089 & 0.084 & 0.047 & 0.316 \\
\hline EL & 0.092 & 0.091 & 0.027 & 0.169 & 0.092 & 0.091 & 0.035 & 0.175 \\
\hline SNN & 0.083 & 0.077 & 0.014 & 0.291 & 0.083 & 0.077 & 0.019 & 0.287 \\
\hline TEL & 0.092 & 0.085 & 0.030 & 0.447 & 0.092 & 0.085 & 0.033 & 0.441 \\
\hline COTE & 0.086 & 0.080 & 0.032 & 0.770 & 0.086 & 0.080 & 0.031 & 0.699 \\
\hline BVB & 0.068 & 0.065 & 0.008 & 0.189 & 0.069 & 0.066 & 0.007 & 0.195 \\
\hline
\end{tabular}




\begin{tabular}{|c|c|c|c|c|c|c|c|c|}
\hline & \multicolumn{9}{|c|}{ FHS_GARCH_DCC } & \multicolumn{3}{c|}{ FHS_GARCH_aDCC } \\
\hline FP & 0.068 & 0.060 & 0.039 & 0.107 & 0.070 & 0.060 & 0.039 & 0.110 \\
\hline TLV & 0.111 & 0.109 & 0.094 & 0.125 & 0.111 & 0.109 & 0.092 & 0.123 \\
\hline SNP & 0.106 & 0.108 & 0.093 & 0.116 & 0.105 & 0.107 & 0.092 & 0.115 \\
\hline SNG & 0.073 & 0.073 & 0.056 & 0.084 & 0.072 & 0.073 & 0.057 & 0.084 \\
\hline BRD & 0.110 & 0.109 & 0.102 & 0.122 & 0.109 & 0.109 & 0.101 & 0.122 \\
\hline TGN & 0.109 & 0.103 & 0.091 & 0.161 & 0.109 & 0.104 & 0.090 & 0.160 \\
\hline EL & 0.073 & 0.068 & 0.063 & 0.089 & 0.073 & 0.067 & 0.063 & 0.089 \\
\hline SNN & 0.098 & 0.107 & 0.059 & 0.121 & 0.099 & 0.107 & 0.059 & 0.124 \\
\hline TEL & 0.090 & 0.090 & 0.081 & 0.104 & 0.091 & 0.091 & 0.080 & 0.103 \\
\hline COTE & 0.113 & 0.103 & 0.073 & 0.183 & 0.110 & 0.100 & 0.070 & 0.188 \\
\hline BVB & 0.048 & 0.049 & 0.037 & 0.056 & 0.051 & 0.050 & 0.040 & 0.058 \\
\hline \hline \multicolumn{8}{|c|}{ FHS_APARCH_DCC } & FHS_APARCH_aDCC \\
\hline FP & 0.060 & 0.053 & 0.033 & 0.091 & 0.063 & 0.055 & 0.033 & 0.098 \\
\hline TLV & 0.113 & 0.115 & 0.091 & 0.135 & 0.112 & 0.114 & 0.093 & 0.133 \\
\hline SNP & 0.108 & 0.110 & 0.089 & 0.124 & 0.107 & 0.109 & 0.089 & 0.120 \\
\hline SNG & 0.080 & 0.084 & 0.065 & 0.093 & 0.079 & 0.083 & 0.063 & 0.092 \\
\hline BRD & 0.108 & 0.106 & 0.094 & 0.127 & 0.108 & 0.106 & 0.094 & 0.126 \\
\hline TGN & 0.112 & 0.108 & 0.088 & 0.161 & 0.112 & 0.107 & 0.090 & 0.160 \\
\hline EL & 0.067 & 0.065 & 0.058 & 0.080 & 0.067 & 0.065 & 0.055 & 0.081 \\
\hline SNN & 0.073 & 0.073 & 0.051 & 0.088 & 0.077 & 0.076 & 0.052 & 0.093 \\
\hline TEL & 0.084 & 0.085 & 0.072 & 0.102 & 0.086 & 0.087 & 0.072 & 0.100 \\
\hline COTE & 0.142 & 0.132 & 0.089 & 0.227 & 0.134 & 0.126 & 0.069 & 0.219 \\
\hline BVB & 0.051 & 0.050 & 0.045 & 0.058 & 0.056 & 0.054 & 0.047 & 0.065 \\
\hline \multicolumn{8}{|c|}{ Source: own computations in R environment } & \\
\hline
\end{tabular}

According to Mina and Xiao (2001) the incremental VaR (also known as component VaR) in its absolute value can be interpreted as the profit and loss for a certain position in the portfolio. Therefore, if a certain sum is invested in stock $\mathrm{j}$, a negative incremental VaR for the same stock is interpreted as a diminishing of the overall risk by the amount or percentage indicated. In this analysis, only the modified VaR approach yielded negative incremental VaR (it can be observed among the minimum values in Table III, at the corresponding method) for certain periods. However, this approach is proven to be inefficient by Martin and Arora (2017) because, as the authors present, the modified VaR estimators yield inflated standard errors.

From the analyzed sample, COTE seems to have the highest contribution while BVB has the lowest. The "shape" of the contributions differs according to the estimation method used. As expected, the models that use a constant variance-covariance matrix have a flatter appearance and the ones with dynamic variance-covariance matrix tend to be more "spiked". The filtered historical simulation approaches are the best at identifying significant structural modifications in the portfolio and the periods with higher risk for certain portfolio components. An investor could use such an analysis in order to adjust the weights of his portfolio components.

The current paper is focused on finding an accurate method for estimating Value-at-risk and to decompose it in order to assess the risk contribution of every asset in the portfolio. The study was conducted on a sample of 11 stocks from the Romanian market.

Another relevant study for VaR estimation is the one proposed by Silahli et al. (2019). Their paper is similar to the present one. They also compare VaR Models that take into account historical simulation, variance-covariance approaches and DCC models. However their best approach is one based on a combination of historical simulation with a GARCH model and innovations that follow a two-sided Weibull distribution, while in our study the best models are the parametric GARCH-aDCC and the FHS-FARCH-DCC. Three of the back testing methods 
used in both, this study and Silahli et al.'s (2019) paper are indentical: Kupiec's (1995)unconditional coverage test, Christofersen's (1998) conditional coverage test and Dynamic Quantile test of Engle and Manganeli (2004).

Another difference between the two studies is that in the present paper filtered historical simulation by Barone-Adesi et al. (1999) is used instead of simple historical simulation. In the right combinations, filtered historical simulation yields some of the best results as proven above in this section.

Silahli et al. (2019) did not take into consideration the use of asymmetric DCC while we explored that possibility. However, they analyze the cryptocurrency market while our study is focused on the stock market which is known for the asymmetry of financial assets returns.

Lu et al. (2014) combined copula methods and GARCH models in order to determine VaR in a Monte Carlo simulation approach. Their dataset consists of oil and natural gas options and futures contracts from the New York Mercantile Exchange during January 1998 - December 2009. They used some of the same tests we use in order to evaluate VaR: Kupiec's (1995) unconditional coverage test, Christofersen's (1998) conditional coverage test and Gonzales-Riviera (2004) loss function. Our study includes asymmetry specific models and equally weighted portfolios, similar to Lu et al.'s (2014). The difference lies in the use of aDCC- GARCH instead of copulas-GARCH classes of models. Also the Monte Carlo simulation is not present in our study.

Other recent studies like Babat et al. (2017) propose an algorithm to help construct a "nearoptimal" VaR portofolio i.e. to allocate the weights optimally. The authors test their method on a sample with 30 to 90 financial assets from the US financial market. Even though the method is very innovative and futuristic, the algorithms are only approximating the optimal VaR as the authors stated. A conservative investor might prefer an approach that allows more manual control over the portfolio allocation, like the ones evaluated in the present study, which were tested on equal weighted portfolios in order to easily observe the risk contribution for every asset individually (from an incremental VaR perspective).

Al Janabi et al. (2019) use a copula based liquidity portfolio VaR measure. Taking liquidity into account provides an advantage due to the fact that it reduces the risk of underestimating the losses as they point out. Their study was conducted on commodities, gold and Bitcoin markets from the G-7 countries group. The present study is different than the study of Al Janabi et al. (2019) since our paper does not take liquidity into account and it was focused only on a frontier stock market, but testing the methods used in this paper on other types of markets could be a subject for a future study.

Jang and Park (2016) present a model which optimizes the portfolio choice taking into account a Value-at-Risk constraint and the concept of model uncertainty. Their complex methodology is more applicable to a fund manager in comparison to the methods presented in this paper which could be used by an investor on the stock market.

Most of the studies referred to in this section do not use the DCC/aDCC approach and none use the combination of filtered historical simulation and the multivariate GARCH we focus on in this paper in order to estimate VaR. In addition, the use of the incremental VaR helps highlight the risk contribution of every financial asset to the overall portfolio. However, the main disadvantage of our paper is the limited data on which the methods were tested. An extension of the data sample could provide a starting point for future research.

\section{Conclusion}

This study focused on filling a small literature gap, in estimating VaR, by the use of multivariate asymmetric DCC models and by combining them with filtered historical simulation for a portfolio of financial assets. The rolling window approach was used in order to make back testing possible. Then a decomposition analysis was performed in order to assess the contribution of every stock to the overall VaR. Ten Value-at-Risk models were analyzed: the first was 
the Riskmetrics approach which was also considered a benchmark, the modified VaR, 4 versions of parametric multivariate $\mathrm{VaR}$ and 4 models using semi-parametric approaches.

The filtered historical simulation methods provided good results in most of the combinations. The best model is the one that uses GARCH for the univariate series and takes asymmetry into account on the multivariate level; this model has the lowest loss from the FHS category.

The modified VaR was better than the benchmark model, but as the results have shown, it overestimates the risk too much. As for the parametric approaches the best one would be GARCH-aDCC.

When it comes to VaR decomposition, all models identify the riskier components in the portfolio, but the FHS approaches also help identify the periods in which a stock presents a higher degree of risk.

The study also has limitations, firstly due to the nature of the data set; enough data could only be obtained for 11 stocks from the Romanian Market between 2014-07-08 and 2019-1004, resulting in 1319 observations. The bootstrap window of 300 observations for the filtered historical simulation approaches is rather small. This method might have yielded better results if it was applied on longer time series. Also, longer data series could prove useful to enlarge the rolling window for all approaches. This could be a subject of a future study.

The VaR models presented here also have practical implementations. One possible application would be for an investor to use the methods discussed in this article for constructing its own portfolio, given the fact that the total possible loss can be decomposed and associated with every single portfolio component.

They can also be used by banks and similar financial entities in order to mitigate their foreign exchange and market risks and even for stress testing. In money-lending institutions the VaR methods could be used for economic capital calculation. The methodologies presented in this paper may have an impact in how financial institutions may set limits (according to their internal policy) with respect to the level of risk they are willing to accept. For example, a financial institution may not accept an investment in a certain asset if its contribution to the overall risk would be higher than an a priori fixed threshold (i.e. no component from the entity's portfolio is allowed to exceed the individual risk threshold). But this is not restricted to individual risks. Overall portfolio limits could also be established.

Regulatory authorities could make use of such Value-at-Risk measures in order to impose thresholds and benchmarks to the financial environment's participants. When it comes to policy, regulators often set limits regarding the amount of risk a financial institution is allowed to take in, but the methods that should be used in order to comply with the restrictions, are not always imposed or specified. Therefore, a financial entity may choose its own. A bank or a similar institution may opt for FHS-GARCH-aDCC in order to manage its current and future market and foreign exchange risks.

\section{REFERENCES}

[1] Abad, P., Benito, S. and Lopez, C. (2014) A comprehensive review of value at risk methodologies, The Spanish Review of Financial Economics, Vol. 12, No. 1, 15-32.

[2] Al Janabi, M. A. M., Ferrer, R., Shahzad, S.J.H., (2019). Liquidity-adjusted value-at-risk optimization of a multi-asset portfolio using a vine copula approach. Physica A, Vol. 536, Article No. 122579. Available on-line at: https://doi.org/10.1016/j.physa.2019.122579.

[3] Artzner, P., Delbaen, F., Eber, J.M. and Heath, D. (1999). Coherent measures of risk. Mathematical Finance, Vol. 9, No.3, 203-228.

[4] Babat, O., Vera, J. C., and Zuloaga, L. F., (2017). Computing near-optimal Value-at-Risk portfolios using Integer Programming techniques. European Journal of Operational Research, Vol 226, No. 1, 304-315.

[5] Banihashemi, S., Navidi, S. (2017). Portfolio performance evaluation in Mean-CVaR framework: A comparison with non-parametric methods value at risk in Mean-VaR analysis. Operations Research Prespective, Vol. 4, 21-28.

[6] Barone-Adesi, G., Giannopoulos, K. and Vosper, L. (1999). VaR without correlations for nonlinear portfolios. Journal of Futures Markets, Vol. 19, No. 5, 583-602. 
[7] Bollerslev, T., (1986). Generalized autoregressive heteroskedasticity. Journal of Econometrics, Vol. 31, No. $3,307-327$.

[8] Boudt, K., Peterson, B. and Croux, C., (2008). Estimation and decomposition of downside risk for portfolios with non-normal returns. Journal of Risk, Vol. 11, No. 2, 79-103.

[9] Cappiello, L., Engle, R.F. and Sheppard, K. (2006). Asymmetric dynamics in the correlations of global equity and bond returns. Journal of Financial Econometrics, Vol. 4, No. 4, 537-572.

[10] Christoffersen, P. (1998). Evaluating interval forecasts. International Economic Review, Vol. 39, No. 4, 841-862.

[11] Daníelsson, J., Jorgensen, B. N., Samorodnitsky, G., Sarma, M., de Vries, C. G. (2012). Fat tails, VaR and subadditivity. Journal of Econometrics, Vol. 172, No. 2, 283-291.

[12] Ding, Z., Granger, C.W.J. and Engle, R.F. (1993). A long memory property of stock market returns and a new model. Journal of Empirical Finance, Vol. 1, No. 1, 83-106.

[13] Engle, R.F. and Sheppard, K. (2001). Theoretical and empirical properties of dynamic conditional correlation multivariate GARCH. NBER Working Paper, available on-line at https://www.nber.org/papers/w8554.pdf.

[14] Engle, R.F. and Manganelli, S. (2004). CaViaR: conditional autoregressive value at risk by regression quantiles. Journal of Business and Economic Statistics, Vol. 22, No. 4, 367-381.

[15] Favre, L. and Galeano. J., (2002). Mean-Modified Value-at-Risk Optimization with Hedge Funds. Journal of Alternative Investment, Vol 5, No. 2, 21-25.

[16] Glasserman, P. (2005). Measuring marginal risk contributions in credit portfolios. Journal of Computational Finance, Vol. 9, No. 2, 1-41.

[17] Glasserman, P., and Li, J. (2005). Importance sampling for portfolio credit risk. Management Science Vol. 51, No. 11, 1643-1656.

[18] Gonzalez-Rivera G., Lee T. H. and Mishra, S. (2004). Forecasting Volatility: A Reality Check Based on Option Pricing, Utility Function, Value-at-Risk, and Predictive Likelihood. International Journal of Forecasting, Vol. 20, No. 4, 629-645.

[19] Hafner, C. M. and Preminger, A. (2010). Deciding between GARCH and stochastic volatility via strong decision rules, Journal of Statistical Planning and Inference, Vol. 140, No. 3, 791-805.

[20] Hallerbach, W. G. (2002). Decomposing Portfolio Value-at-Risk: A General Analysis. Journal of Risk, Vol. 5, No. 2, 1-18.

[21] Halkos, G. E., Tsirivis, A. S. (2019). Value-at-risk methodologies for effective energy portfolio risk management. Economic Analysis and Policy, Vol. 62, 197-212.

[22] Jain, S. and Chakrabarty, S. P. (2019). Does Marginal VaR Lead to Improved Performance of Managed Portfolios: A Study of S\&P BSE 100 and S\&P BSE 200. Asia-Pacific Financial Markets, available online at: https://doi.org/10.1007/s10690-019-09294-0.

[23] Jang, B-G., Park, S. (2016). Ambiguity and Optimal Portfolio Choice with Value-at-Risk Constraint. Finance Research Letters, Vol 18, 158-176.

[24] Kellner, D. and Rösch, D. (2016). Quantifying market risk with Value-at-Risk or Expected Shortfall? Consequences for capital requirements and model risk. Journal of Economic Dynamics and Control, Vol. $68,45-63$

[25] Kim, T., Manganelli, S. and White, H. (2015). VAR-for-VaR: Measuring Tail Dependence Using Multivariate Regression Quantiles. Journal of Econometrics, Vol. 187, No. 1, 169-188.

[26] Krause, J. and Paolella, M. S. (2014). A Fast, Accurate Method for Value-at-Risk and Expected Shortfall, Econometrics, Vol. 2, No. 2, 98-122; available on-line at https://doi.org/10.3390/econometrics2020098.

[27] Kupiec, P. H. (1995). Techniques for verifying the accuracy of risk measurement models. Journal of Derivatives, Vol. 3, No. 2, 73-84.

[28] Le, T.H. (2020). Forecasting value at risk and expected shortfall with mixed data sampling International Journal of Forecasting, available on-line at https://doi.org/10.1016/j.ijforecast.2020.01.008.

[29] Lu, X. F., Lai, K. K., and Liang, L. (2014). Portfolio value-at-risk estimation in energy futures markets with time-varying copula-GARCH model. Annals of Operation Research, Vol. 219, 333-357.

[30] Martellini, L. and Ziemann, V. (2010). Improved Estimates of Higher-Order Comoments and Implications for Portfolio Selection. The Review of Financial Studies, Vol. 23, No. 4, 1467-1502.

[31] Martin, R..D. and Arora, R. (2017). Inefficiency and bias of modified value-at-risk and expected shortfall. Journal of Risk, Vol. 19, No. 6, 59-84.

[32] Mina, J. and Xiao, J. Y. (2001), Return to RiskMetrics: The Evolution of a Standard. New York, available online at: https://www.msci.com/documents/10199/dbb975aa-5dc2-4441-aa2d-ae34ab5f0945.

[33] Morgan, J.P., (1996). Riskmetrics Technical Document, 4th ed. J.P. Morgan, New York.

[34] Rau-Bredow, H. (2002). Value at Risk, Expected Shortfall, and Marginal Risk Contribution, in Szego, G., Risk Measures for the 21st Century, pp. 61-68, Wiley.

[35] Silahli, B., Dingec K. D., Cifter, A., Aydin, N. (2019). Portfolio value-at-risk with two-sided Weibull distribution: Evidence from cryptocurrency markets. Finance Research Letters, available on-line at: https://doi.org/10.1016/j.frl.2019.101425. 
[36] Su, Q., Qin, Z., Peng, L., Qin, G., (2020). Efficiently Backtesting Conditional Value-at-Risk and Conditional Expected Shortfall, Journal of the American Statistical Association, available on-line at https://doi.org/10.1080/01621459.2020.1763804.

[37] Taylor, J.W. (2020), Forecast combinations for value at risk and expected shortfall, International Journal of Forecasting, Vol. 36, No. 2, 428-441.

[38] Yamai, Y. and Yoshiba, T. (2002), Comparative Analyses of Expected Shortfall and Value-at-Risk: Their Estimation Error, Decomposition, and Optimization. Monetary and Economic studies, Vol. 20, No. 1, 87121. 
6. Appendix 1: List of COMPanies included in the Study

\begin{tabular}{|l|l|l|}
\hline Symbol & \multicolumn{1}{|c|}{ Name } & \multicolumn{1}{|c|}{ Sector of activity } \\
\hline BRD & BRD - GROUPE SOCIETE GENERALE S.A. & Banking \\
\hline BVB & BURSA DE VALORI BUCURESTI S.A. & Financial sector \\
\hline COTE & CONPET S.A. & Natural gas industry \\
\hline EL & SOCIETATEA ENERGETICA ELECTRICA S.A. & Energy industry \\
\hline FP & FONDUL PROPRIETATEA & Financial sector \\
\hline SNG & S.N.G.N. ROMGAZ S.A. & Natural gas industry \\
\hline SNN & S.N. NUCLEARELECTRICA S.A. & Energy industry \\
\hline SNP & OMV PETROM S.A. & Oil industry \\
\hline TEL & C.N.T.E.E. TRANSELECTRICA & Energy industry \\
\hline TGN & S.N.T.G.N. TRANSGAZ S.A. & Natural gas industry \\
\hline TLV & BANCA TRANSILVANIA S.A. & Banking \\
\hline
\end{tabular}

7. 
8. Appendix 2: Contribution to VaR in percentages

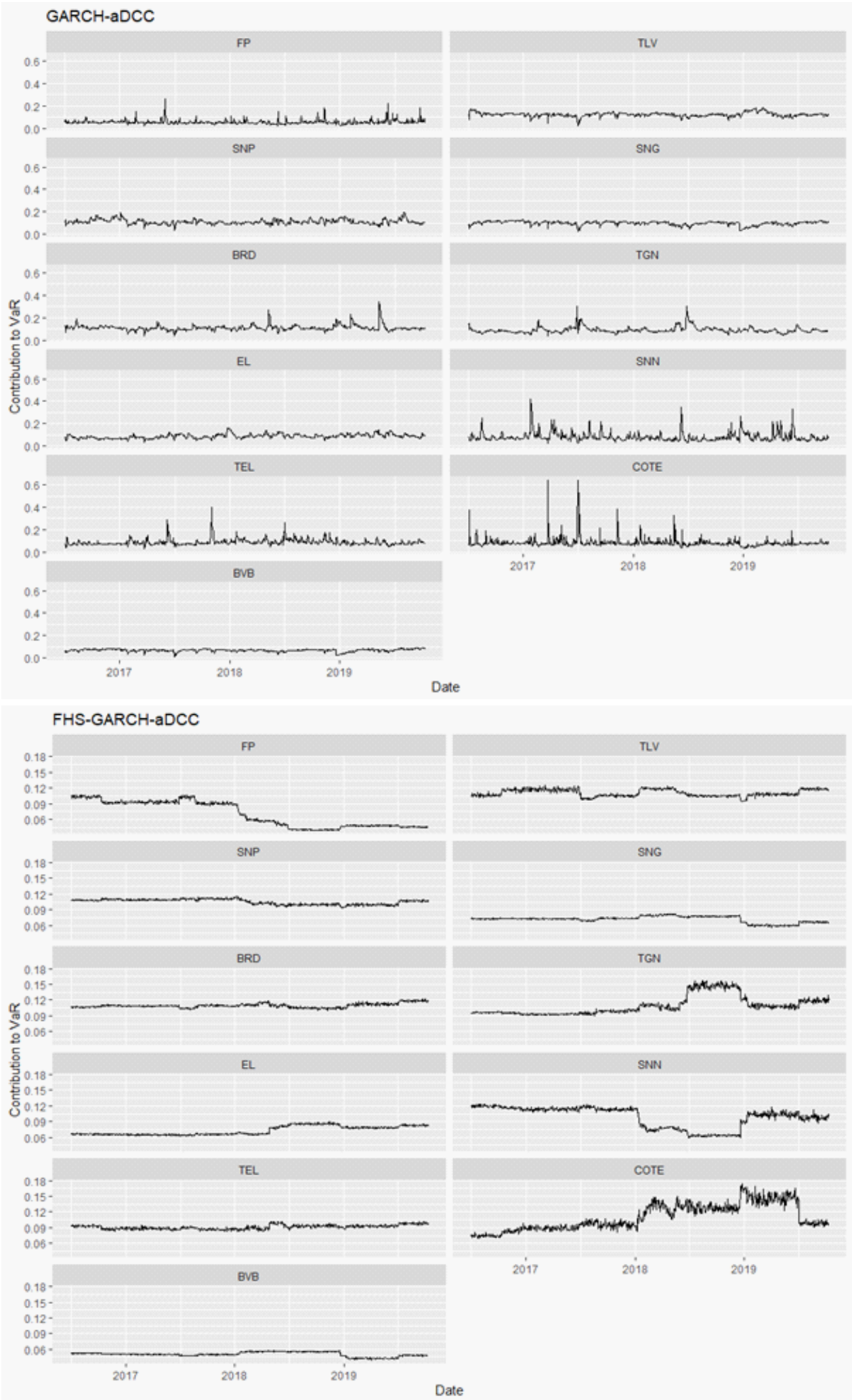

